\title{
Comments on "Review of the association between periodontitis and chronic obstructive pulmonary disease in smokers"
}

\author{
Irineu Gregnanin Pedron \\ Department of Periodontology, Implantology and Stomatology, Universidade Brasil, São Paulo, $1^{\text {st }}$ Lieutenant of \\ Brazilian Air Force, Hospital de Força Aérea de São Paulo, Brazil
}

Dear Editor,

I have read, with great enthusiasm, the article entitled "Review of the association between periodontitis and chronic obstructive pulmonary disease in smokers", authored by Agathi Spiropoulou and colleagues [1], published in issue 1 of the $89^{\text {th }}$ volume (2019) of the Monaldi Archives for Chest Disease. The article is very interesting and reviews the association between periodontitis and chronic obstructive pulmonary disease in smokers. I congratulate the authors Spiropoulou and colleagues for the article presented. However, I would highlight some important points:

Periodontal Medicine is a branch of Periodontal Science that deals with periodontal infection as a possible risk factor for other diseases and / or systemic conditions and their relationships. It was based on the Theory of Focal Infection proposed by Rush and Hunter in the early nineteenth century, but we can consider the postulations of Hippocrates as early as the Old Age [2].

It is important to emphasize that Periodontal Medicine does not invalidate the principle of the traditional relationship between oral manifestations resulting from systemic diseases, but brings a new view of oral diseases, particularly periodontal diseases, considered as infectious foci, which may be a factor risk in relation to other diseases or other systemic conditions. Thus, there are interactions between periodontology and some medical specialties, such as cardiology, neurology, gynecology and obstetrics, endocrinology, pneumology and otorhinolaryngology [2].

In cardiology, the relationship between infectious endocarditis (by Streptococcus viridans) and its origin in the oral cavity has been demonstrated, as well as the possibility of invasive dental procedure

\footnotetext{
Correspondence: Irineu Gregnanin Pedron, Rua Flores do Piauí 508, São Paulo 08210-200, Brazil.

Tel. +55.11.2944-4067.

E-mail: igpedron@alumni.usp.br
}

Key words: Periodontal diseases; periodontics; lung diseases; cardiovascular diseases.

Received for publication: 24 August 2019.

Accepted for publication: 2 September 2019.

${ }^{\mathbb{C}}$ Copyright: the Author(s), 2019

Licensee PAGEPress, Italy

Monaldi Archives for Chest Disease 2019; 89:1157

doi: 10.4081/monaldi.2019.1157

This article is distributed under the terms of the Creative Commons Attribution Noncommercial License (by-nc 4.0) which permits any noncommercial use, distribution, and reproduction in any medium, provided the original author(s) and source are credited. as a risk factor in individuals susceptible to infections. Recently, atherosclerosis has been identified as a disease of infectious origin. Periodontal infection could increase bacteremia and leukocytosis, exposing the host to lipopolysaccharides, Gram-negative microorganisms (such as Porphyromonas gingivalis, Aggregatibacter actinomycetencomitans, Tannerella forsythia and Prevotella intermedia), inflammatory mediators (prostaglandins, interleukose and TNF- $\alpha$ ) and cytokines, which could affect endothelial integrity, promoting changes in plasma lipoprotein metabolism, vascular degeneration, platelet aggregation, and blood coagulation (with high fibrinogen levels), initiating or exacerbating atherogenesis and other thromboembolic events. Not only cardiovascular changes would be consequent, but also stroke [2].

Obstetric complications can be triggered or stimulated by periodontal infections. Studies of preterm birth, low birth weight and abortion have been demonstrated in hamsters. The suggested mechanism was the existence of high levels of prostaglandin E2 in the gingival fluid of pregnant patients with obstetric alterations, associated with subclinical bacteremia resulting from periodontal infection, which may trigger an inflammatory process in the chorionic-amniotic membranes [2].

The relationship between periodontal disease and diabetes is bilateral. Diabetic patients are at higher risk of developing infections and peripheral vasculopathies such as periodontal disease, which in turn makes it difficult to maintain normoglycemia. High levels of cytokines due to subclinical bacteremia may cause complications in diabetic patients [2].

In the respiratory tract, periodontal disease would function as a possible reserve of microorganisms in the oral cavity and oropharynx, aspiration being the main route of contamination, not excluding, however, hematogenous dissemination by contiguity or direct inoculation (traumatic or surgical). Other pulmonary pathologies were also related, beyond the chronic obstructive pulmonary disease (COPD) [1,2], pulmonary emphysema, septic pulmonary embolism, pulmonary empyema or abscesses, and cystic fibrosis. Rhinitis and sinusitis have also been related, and periodontal disease is an important risk factor due to tissue contiguity [2]. It is worth noting that from this perspective of Periodontal Medicine, having periodontal infection as a risk factor to systemic conditions, the susceptibility of the organism must also be considered [2].

More recently, other lines of research in Periodontal Medicine have been approached, such as the relationship between periodontal diseases and obesity, dermatological changes (alopecia), infections in orthopedic appliances and prostheses, other endocrinological complications (thyroid disorders), and Alzheimer's disease, among others [2].

Periodontal treatment resulted in the reduction of serum inflammatory biomarkers, indicating that treatment of periodontal disease may improve systemic activity. Periodontal treatment reduced the 
levels of C-reactive protein, TNF $\alpha$, interleukin-6, leukocytes and fibrinogen, showing beneficial effects on these biochemical parameters considered representative of cardiovascular risk [3].

The presence of the dentist in the Intensive Care Unit (ICU) for preventive and curative procedures (when necessary) has several benefits for hospitalized patients and, subsequently, for public health. Considering nosocomial pneumonia, it is necessary, on average, 10 days of hospitalization and the administration of two distinct antibiotics. In a prospective evaluation, it is assumed that the preventive performance of the dental surgeon through clinical procedures and oral hygiene guidance reduces approximately 4 days of hospitalization and $40 \%$ of the mortality rate. In this perspective, if we take for example a medium-sized hospital (8 to 10 ICU beds), such as ours, the average reduction of $€ 450,000$ per year is estimated.

Ten years ago, at the Air Force Hospital of São Paulo, of the Brazilian Air Force, I work, in addition to my duties as a clinical periodontist and military, as a dentist working in the beds of the inpatient units and in the ICU, practicing preventive dentistry and, when necessary, curative, with clinical and especially periodontal procedures.

Additionally, it is worth emphasizing the importance of antismoking campaigns, avoiding the double etiological factor of periodontal diseases and COPD, as demonstrated by Spiropoulou et al. [1].

Future studies are needed for the qualitative and quantitative assessment of the quality of life of hospitalized patients, as well as the cost savings in public health, based on preventive and curative hospital dental practice.

\section{References}

1. Spiropoulou A, Zareifopoulos N, Bellou A, et al. Review of the association between periodontitis and chronic obstructive pulmonary disease in smokers. Monaldi Arch Chest Dis 2019; 89:1018.

2. Pedron IG. Periodontal medicine. In: Varellis MLZ, editor. The patient with special needs in dentistry - Practical guide. São Paulo: Santos; 2017; pp 477-8.

3. Roca-Millan E, González-Navarro B, Sabater-Recolons MM, et al. Periodontal treatment on patients with cardiovascular disease: Systematic review and meta-analysis. Med Oral Patol Oral Cir Bucal 2018;23:e681-90. 